\title{
Structure and Short Range Order of Two Phases in the Single Domain $\mathrm{Mn}_{0.75} \mathrm{Cu}_{0.25}$ Alloy after Spinodal Decomposition
}

\author{
J. Jankowska-KisielińskA*, K. Świderska And K. Mikke \\ Institute of Atomic Energy, 05-400 Otwock-Świerk, Poland
}

\begin{abstract}
The aim of the present paper was to identify the atomic and magnetic structure and short-range order of the two phases present in the $\mathrm{Mn}_{0.75} \mathrm{Cu}_{0.25}$ alloy after decomposition and plastic deformation. The sample was investigated by elastic neutron scattering at small-angle and 3-axes spectrometers. We have confirmed the decomposition of the alloy into two types of regions with manganese concentration increased and decreased as compared with the mean value. The crystal lattice in regions of both types is tetragonal (pseudo FCT) below $T_{\mathrm{N}}$ but degree of tetragonal distortion is different. The long range antiferromagnetic order is present only in the regions of bigger distortion and higher $\mathrm{Mn}$ concentration. In the regions of lower Mn content and smaller distortion there is a short range ordering of complex magnetic and atomic character.
\end{abstract}

PACS numbers: 61.05.F-, 75.30.Fv, 75.40.-s, 75.50.Lk

\section{Introduction}

$\mathrm{Mn}_{x} \mathrm{Cu}_{1-x}$ alloys with manganese concentration above $x=0.5$ reveal a tendency to composition segregation. In these alloys spinodal decomposition occurs in the temperature range 650-900 $\mathrm{K}$ resulting in a formation of regions of two types with different manganese concentration, increased and decreased as compared with the average value $[1,2]$.

The aim of the present work was to identify the lattice and magnetic structure of the two phases formed in the $\mathrm{Mn}_{0.75} \mathrm{Cu}_{0.25}$ alloy after spinodal decomposition and plastic deformation.

The aging process is expected to cause the decomposition of the homogeneous $\mathrm{Mn}_{0.75} \mathrm{Cu}_{0.25}$ alloy into regions with the manganese content $x \approx 0.9$ and $x \approx 0.4[1,2]$. The subsequent plastic deformation makes the sample single-domain but it can change structural, magnetic and mechanical properties of the sample. We wanted to test, to what extent the both regions of the decomposed sample preserve the structural properties of the single-phase samples with similar manganese content.

Alloys with low manganese concentration $(x \leq 0.4)$ tend to form atomic short range arrangement and magnetic short range ordering of incommensurate spin density wave (ISDW) type [3]. In earlier investigations on the $\mathrm{Mn}_{0.4} \mathrm{Cu}_{0.6}$ alloy the magnetic short range order of the ISDW type was confirmed $[4,5]$.

In quenched alloys with $x>0.7$ and aged alloys with $x \geq 0.5$ there is an antiferromagnetic long range ordering

\footnotetext{
* corresponding author; e-mail:

j.jankowska_kisielinska@cyf.gov.pl
}

of AF1 type [6]. Phase transition from the paramagnetic to the AF1 phase is accompanied by tetragonal crystal lattice deformation.

The present investigation of the $\mathrm{Mn}_{0.75} \mathrm{Cu}_{0.25}$ alloy after aging and plastic deformation started with confirmation, by small-angle neutron scattering (SANS), of the spinodal decomposition. The elastic neutron scattering by 3 -axes spectrometer was used for further investigations. It was revealed that regions of both types are characterized by different degree of tetragonal distortion. This fact enables us to attribute the observed neutron scattering to regions of the appropriate type. Antiferromagnetic long-range ordering is present in the regions with elevated manganese content. In the regions of the second type there is a short-range ordering which is the cause of diffuse neutron scattering observed in vicinity of the $(0.5,0,1)$ reciprocal lattice point (RLP). Our investigations of the temperature dependence of those effects revealed complex magnetic and atomic nature of the short-range order in manganese depleted regions.

\section{Investigation of decomposition and structure of decomposed alloy}

The investigated sample of the $\mathrm{Mn}_{0.75} \mathrm{Cu}_{0.25}$ alloy is a single crystal with the mosaic spread $\eta=150^{\prime}$ and is nearly cubic of the volume $1 \mathrm{~cm}^{3}$. The sample was warmed at temperature $\approx 1100 \mathrm{~K}$ and quenched to $295 \mathrm{~K}$ in order to obtain the metastable $\gamma$-phase of the alloy. Then it was aged at $\approx 720 \mathrm{~K}$ with the aim to cause the spinodal decomposition of the concentration and quenched again to $295 \mathrm{~K}$. Subsequently it was deformed by compression along 3 possible directions of the [100] type. Compression was performed under control of deformation up to $4 \%$. 


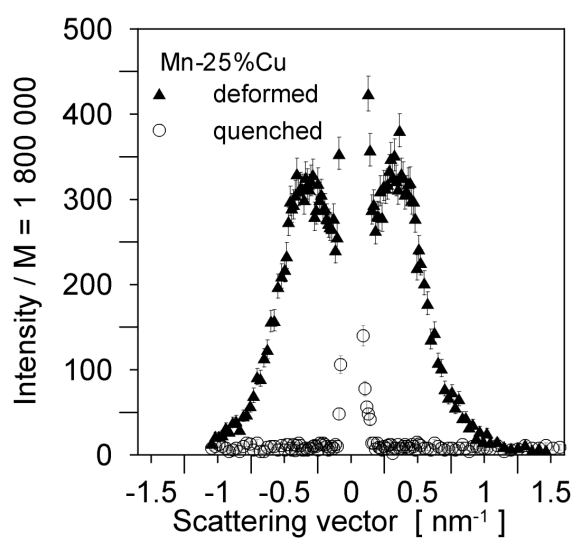

Fig. 1. Results of the SANS measurements for the decomposed sample of $\mathrm{Mn}_{0.75} \mathrm{Cu}_{0.25}$ alloy and for the quenched sample of the same mean composition.

The effects of these thermal and plastic processes were then tested by SANS spectrometer at MARIA reactor in IAE, Swierk. The results (Fig. 1) confirm the spinodal decomposition with the periodicity about $18-20 \mathrm{~nm}$. Thus the dimensions of the regions of the two types are about 9-10 nm.

Crystal lattice structure was tested by neutron scattering at the Bragg reflexes (200) and (002) at triple axes spectrometer (Fig. 2a,b). The neutron wavelength was $0.235 \mathrm{~nm}$. The spectrometer resolution for a longitudinal scan at the $(200)$ RLP is $\Delta q \cong 0.55 \mathrm{~nm}^{-1} \cong 0.033 \times 2 \pi / a$. For the scattering vector perpendicular to the direction of the last deformation we have obtained the strong reflex (200) for the wave-vector length $4 \pi / a$ and the weak reflex for the length $4 \pi / c 1$. For the scattering vector parallel to the direction of the last deformation (Fig. 2b) the splitting of the observed intensity distribution into component reflexes is not so obvious. It is possible to distinguish 4 reflexes: two strong reflexes (002) for vector lengths $4 \pi / c 1$ and $4 \pi / c 2$ and two weak reflexes, one for vector length $4 \pi / a$, and the second one probably spurious. The obtained positions of the maxima of weak reflexes are not fully reliable, but their inaccurate definition does not influence much the obtained positions of the maxima of strong reflexes. Diffuse magnetic scattering observed in the narrow range of the scattering-vector length close to the $2 \pi / c 1$ at the (001) RLP was used for more precise estimation of the value of $c 1$.

The following mean values of lattice constants were obtained from the maxima positions at $15 \mathrm{~K}$ : $a=$ $0.3747 \pm 0.0001 \mathrm{~nm}, c 1=0.3600 \pm 0.0002 \mathrm{~nm}$ (tetragonal deformation $1-c 1 / a=0.03932 \pm 0.00002)$ and $c 2=0.3678 \pm 0.0014 \mathrm{~nm}$ (tetragonal deformation $1-$ $c 2 / a=0.01848 \pm 0.00007)$.

The widths of the observed maxima are of the order of $\approx(0.05-0.62) \pi / a$ while the spectrometer resolution for a longitudinal scan: $\Delta q \cong 0.033 \times 2 \pi / a$. The broadening is due probably to the internal stresses and concentration inhomogeneity inside each phase. Deviation of the lattice

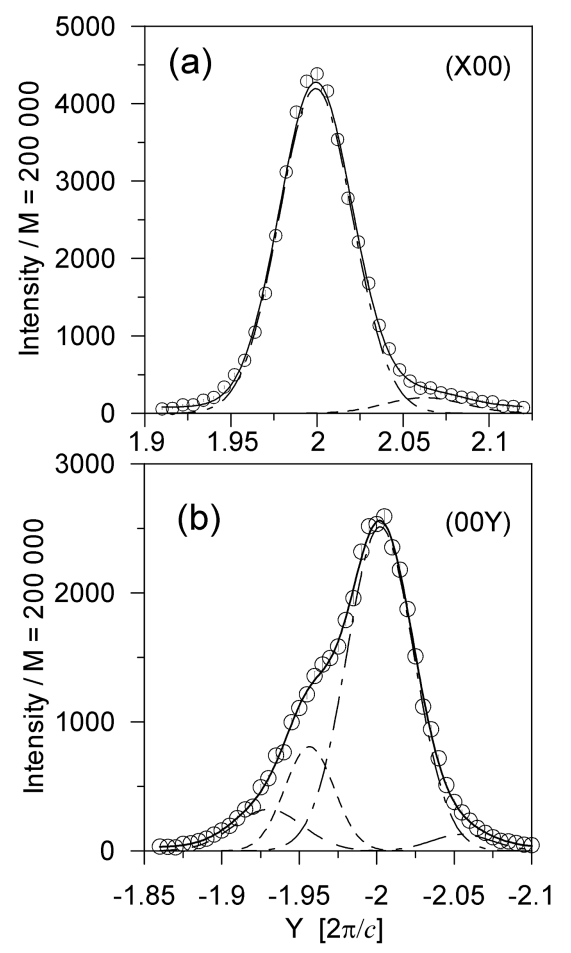

Fig. 2. Distributions of the elastic neutron scattering intensity in the proximity of the (200) RLP - (a) and that of the (002) RLP - (b), measured at $15 \mathrm{~K}$. The solid lines represent the best fit results computed as a sum of the Gaussian distributions, and the dashed lines are their components.

constant is $0.008-0.010 \mathrm{~nm}$ for $a$ and $0.010-0.012 \mathrm{~nm}$ for $c 1$ and $c 2$.

The positions in the reciprocal space of the magnetic (110) and (201) Bragg reflexes indicate that the sample is antiferromagnetic of the AF1-type. Distribution of the magnetic domains was obtained by measuring of the intensity of the (110)-type Bragg reflexes. The majority of domains $(82 \pm 4 \%)$ have their magnetic moments parallel to the direction of the last deformation, say [001], other domains $(12 \pm 3 \%$ and $6 \pm 2 \%)$ have their moments parallel to the [100] and [010] direction, respectively.

The Nèel temperature of the manganese-rich regions of the sample, $T_{\mathrm{N}}=(455 \pm 5) \mathrm{K}$ is estimated on the basis of the temperature dependence of the intensity of (201) and (110) magnetic Bragg reflexes measured in the temperature range $350-480 \mathrm{~K}$.

\section{Neutron diffuse scattering measurements as a function of temperature}

The essential part of measurements applied to diffuse scattering in the vicinity of the $(1,0,0.5)$ and $(0.5,0,1)$ RLP. The measurements were performed in the temperature range $15-290 \mathrm{~K}$ at the 3 -axis spectrometer. The pyrolytic graphite monochromator and analyzer was used. The wavelength of scattered neutrons was $\lambda=0.235 \mathrm{~nm}$. 
The application of $6 \mathrm{~cm}$ pyrolytic graphite filter enabled to exclude neutrons of higher order reflexes of monochromator with wavelengths $\lambda / 2$ and $\lambda / 3$. The measurements were performed in the $(010)$ plane in which the [001] vector was parallel to the last deformation direction and the [100] vector was perpendicular to that direction.

Figure 3 illustrates the temperature dependence of lattice constants which were determined on the basis of position of various diffraction peaks. Lattice constants values indicate that diffuse scattering in the vicinity of the $(0,0,1)$ RLP is related to sample regions with lattice constants $a$ and $c 1$, whereas diffuse scattering in the vicinity of $(1,0,0.5)$ and $(0.5,0,1)$ lattice points is associated with regions with lattice constants $a$ and $c 2$. In this aspect our results are in agreement with the results obtained for the undeformed $\mathrm{Mn}_{0.82} \mathrm{Cu}_{0.18}$ alloy sample [7].

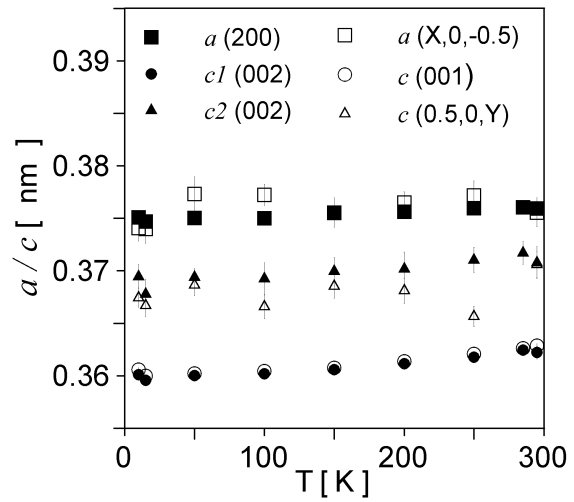

Fig. 3. Temperature dependence of lattice constant values obtained from Bragg peak positions: $a(2,0,0)$, $c 1(0,0,2)$ and $c 2(0,0,2)$, and those obtained from diffuse scattering: $c(0,0,1), a(x, 0,0.5)$, and $c(0.5,0, y)$.

Intensity distributions of the diffuse scattering centred at the $(0.5,0,-1)$ RLP for [100] and [001] directions (examples of which are presented in Figs. 4 and 5 for a few temperature values) indicate that temperature change is followed by changes in both intensity and shape of distributions.

Intensity decrease connected with temperature increase may suggest a magnetic character of scattering, but it may be partially caused by changes of the DebyeWaller factor value. Figure 6 illustrates the temperature dependence of integrated intensity of the Bragg scattering in the vicinity of the (200) RLP, magnetic Bragg scattering in the vicinity of the (201) RLP, and diffuse scattering in the vicinity of the $(0.5,0,1) \mathrm{RLP}$, for the present sample compared to that for the $\mathrm{Mn}_{0.4} \mathrm{Cu}_{0.6}$ alloy. The intensities are normalized to 1 for the lowest temperature.

The origin of reflexes (200) and (002) is of atomic nature and their dependence on the temperature is due to Debye-Waller factor. Temperature dependence of scattering intensities of peaks mentioned above indicates that in the investigated alloy the Debye-Waller factor affects scattered intensities negligibly up to $250 \mathrm{~K}$. Then the in-

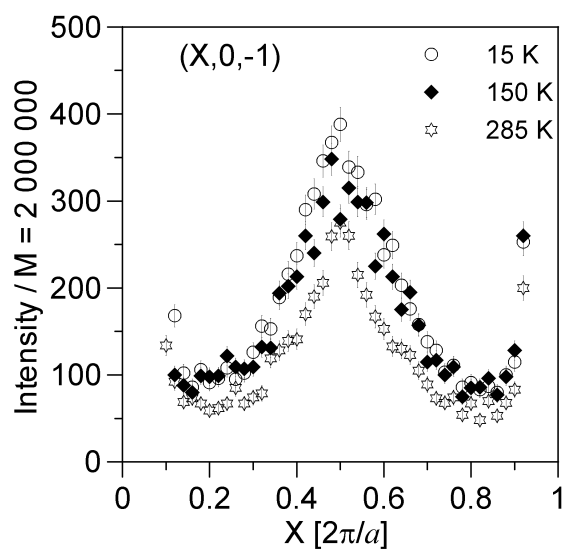

Fig. 4. The intensity distribution of elastically scattered neutrons in the [100] direction, in the vicinity of $(0.5,0,-1)$ RLP, for a few temperature values.

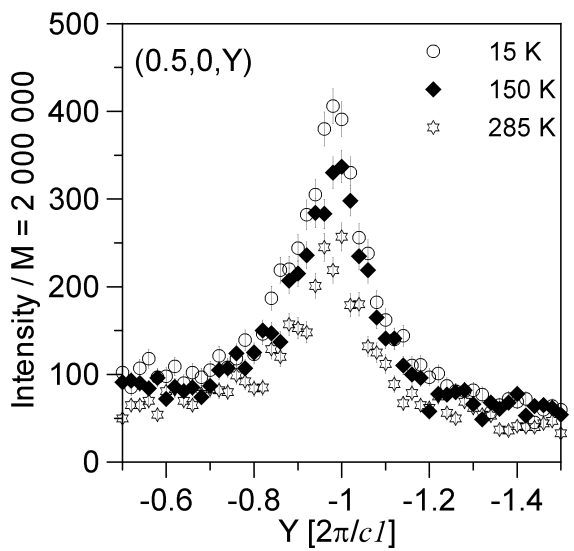

Fig. 5. The intensity distribution of elastically scattered neutrons in the vicinity of $(0.5,0,-1)$ RLP, in the [001] direction, for a few temperature values.

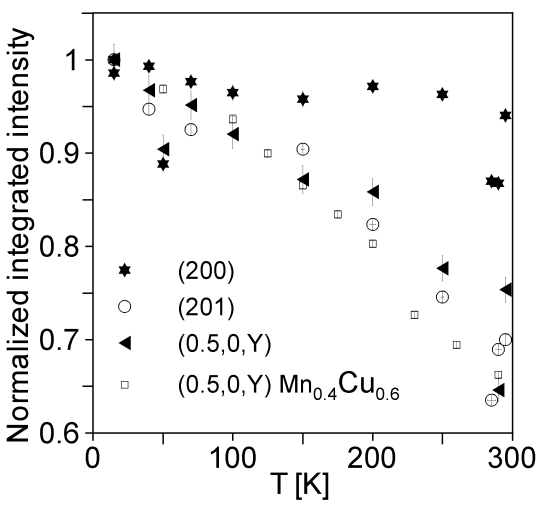

Fig. 6. Temperature dependence of normalized integrated intensity of Bragg scattering in the vicinity of the (200) RLP, magnetic Bragg scattering in the vicinity of the (201) RLP, and diffuse scattering in the vicinity of the $(0.5,0,1)$ RLP for the present sample and that for the $\mathrm{Mn}_{0.4} \mathrm{Cu}_{0.6}$ alloy. 
tensity decreases, reaching $90 \%$ of its initial value at the temperature of $290 \mathrm{~K}$. Magnetic scattering intensity observed at the (201) RLP decreases, while the temperature increases from $10 \mathrm{~K}$ to $290 \mathrm{~K}$, reaching $65 \%$ of its initial value. The intensity of diffuse scattering at the $(0.5,0,1)$ RLP decreases visibly faster than the intensity of nuclear scattering but not as fast as the magnetic one, as the temperature is elevated. The temperature dependence of diffuse scattering intensity for the present sample is also not as pronounced as that for the $\mathrm{Mn}_{0.4} \mathrm{Cu}_{0.6}$ alloy [5].

\section{Discussion and conclusions}

We have confirmed the decomposition of the sample of the $\mathrm{Mn}_{0.75} \mathrm{Cu}_{0.25}$ alloy into regions of two phases with manganese concentration increased and decreased as compared to the mean value. The crystal lattice of both phases is tetragonal with $c / a<1$ below $T_{\mathrm{N}}$ (pseudo FCT) with different tetragonal distortion. The value of the tetragonal distortion parameter $(1-c / a)$ of one phase is more than twice bigger than that of the second one. The lattice distortion connected to the atomic short-range order was reported earlier in $\mathrm{Mn}_{x} \mathrm{Cu}_{1-x}$ alloys for $x=0.35$ and $x=0.23$, but $c / a>1$ [7] contrary to present case. Probably distortion of the manganese poor phase in the decomposed sample is caused by internal stresses introduced by plastic deformation.

The long-range antiferromagnetic order of AF1 type is present only in the regions of the sample with the mean value of the lattice constants $a$ and $c 1$. Reflexes (201) characteristic for this structure have maxima in positions characterised by the lattice constant $a$ in [100] direction and lattice constant $c 1$ in [001] direction, but not in the positions characterised by the lattice constant $c 2$ in the [001] direction. Diffuse magnetic scattering at the (001) RLP originates from the regions with the same constant. Therefore we conclude that regions characterised by the constants $a$ in [100] direction and $c 2$ in [001] direction have not antiferromagnetic order of AF1 type. Reflexes (101) observed in the scattering plane have their maxima in positions characterised by lattice constant $a$ in both [100] and [001] directions. They are interpreted as coming from the minority domains of regions with long range AF1-type ordering. It is interesting that the lattice constant $a$ of these domains is not distorted for the [001] direction of the last deformation. The magnetic ordering and bigger tetragonal distortion allow us to identify the phase characterised by lattice constants $a$ and $c 1$ as the phase of higher manganese content.

The diffuse neutron scattering centred in the proximity of the $(0.5,0,-1)$ RLP has its maximum at the position characterised by the $c 2$ lattice periodicity in the [001] direction, the maximum position along [100] direction is badly defined. The diffuse scattering centred at
$(1,0,-0.5)$ RLP has the maximum at the position characterised by the lattice periodicity $a$ along [100] direction. We conclude that diffuse scattering centred at the $(1,0,0.5)$ RLP and symmetry related points comes from the regions characterised by the $c 2$ lattice periodicity in the direction parallel to the last deformation and the $a$ periodicity in the perpendicular direction. This type of short-range order indicates that phase characterised by $a$ and $c 2$ is the phase of lower manganese content.

The temperature dependence of the intensity of the diffuse scattering centred at the $(1,0,0.5)$ RLP and symmetry related points indicates that at least part of the scattering has magnetic character. The last statement contradicts earlier results for monocrystalline samples of $\mathrm{Mn}_{0.85} \mathrm{Cu}_{0.15}$ [8] and $\mathrm{Mn}_{0.82} \mathrm{Cu}_{0.18}$ [9] alloy that diffuse scattering in the vicinity of the $(0.5,0,1)$ RLP has nonmagnetic character. However, the temperature dependence of the diffuse scattering intensity has not been investigated below $290 \mathrm{~K}$ for any of these cases. We have compared the temperature dependence of the diffuse scattering of the present sample with that for the $\mathrm{Mn}_{0.4} \mathrm{Cu}_{0.6}$ alloy in the temperature range $15-290 \mathrm{~K}$. Magnetic character of the part of the diffuse scattering in the $\mathrm{Mn}_{0.4} \mathrm{Cu}_{0.6}$ alloy was shown earlier [5]. The temperature dependence of the intensity in that sample is more pronounced than in the present decomposed sample. In the present sample the magnetic component is probably lower than in the previous one.

In conclusion the antiferromagnetic ordering of the AF1 type is present only in the manganese-rich phase of the decomposed sample. In the manganese-poor phase there is only short-range order of the complex atomic and magnetic nature.

\section{References}

[1] J.H. Smith, E.R. Vance, J. Appl. Phys. 40, 4853 (1969).

[2] E.Z. Vintaikin, V.B. Dmitriev, V.A. Udovenko, Fiz. Met. Metallov. 46, 790 (1978).

[3] J.W. Cable, S.A. Werner, G.P. Felcher, N. Wakabayashi, Phys. Rev. Lett. 49, 829 (1982).

[4] T.M. Harders, P. Wells, J. Phys. F, Met. Phys. 13, 1017 (1983).

[5] J. Jankowska-Kisielińska, K. Mikke, I. Fijał-Kirejczyk, Acta Phys. Pol. A 113, 1171 (2008).

[6] G.E. Bacon, I.W. Dunmur, J.H. Smith, R. Street, Proc. R. Soc. A 241, 223 (1957).

[7] Y. Tsunoda, J.W. Cable, Phys. Rev. B 46, 930 (1992).

[8] Y. Shiozaki, Y. Nakai, J. Phys. Soc. Japan 47, 817 (1979).

[9] E.Z. Vintaikin, V.B. Dmitriev, V.A. Udovenko, Fiz. Met. Metallov. 59, 742 (1985). 